\title{
From Experimental Data to Pole Parameters in a Model Independent Way
}

\author{
Alfred Švarc* \\ Rudjer Bošković Institute, Bijenička cesta 54, P.O. Box 180, 10002 Zagreb, Croatia \\ E-mail: svarceirb.hr
}

It is well known that unconstrained single-energy partial wave analysis (USEPWA) gives many equivalent discontinuous solutions, so a constraint to some theoretical model must be used to ensure the uniqueness. It can be shown that it is a direct consequence of not specifying the angle-dependent part of continuum ambiguity phase which mixes multipoles, and by choosing this phase we restore the uniqueness of USEPWA, and obtain the solution in a model independent way. Up to now, there was no reliable way to extract pole parameters from so obtained SE partial waves, but a new and simple single-channel method (Laurent + Pietarinen expansion) applicable for continuous and discrete data has been recently developed. It is based on applying the Laurent decomposition of partial wave amplitude, and expanding the non-resonant background into a power series of a conformal-mapping, quickly converging power series obtaining the simplest analytic function with well-defined partial wave analytic properties which fits the input. The generalization of this method to multi- channel case is also developed and presented. Unifying both methods in succession, one constructs a model independent procedure to extract pole parameters directly from experimental data without referring to any theoretical model.

XVII International Conference on Hadron Spectroscopy and Structure - Hadron 2017

25-29 September, 2017

University of Salamanca, Salamanca, Spain

${ }^{*}$ Speaker. 


\section{Introduction}

It is well known that unconstrained single-energy partial wave analysis (USEPWA) gives many equivalent discontinuous solutions, so a constraint to some theoretical model must be used to ensure the uniqueness. It can be shown that it is a direct consequence of not specifying the angle-dependent part of continuum ambiguity phase which mixes multipoles, and by choosing this phase we restore the uniqueness of USEPWA, and obtain the solution in a model independent way [1]. Up to now, there was no reliable way to extract pole parameters from so obtained SE partial waves, but a new and simple single-channel method (Laurent + Pietarinen expansion) applicable for continuous and discrete data has been recently developed [2, 3, 4]. It is based on applying the Laurent decomposition of partial wave amplitude, and expanding the non-resonant background into a power series of a conformal-mapping, quickly converging power series obtaining the simplest analytic function with well-defined partial wave analytic properties which fits the input. The method is particularly useful to analyse partial wave data obtained directly from experiment because it works with minimal theoretical bias since it avoids constructing and solving elaborate theoretical models, and fitting the final parameters to the input, what is the standard procedure now. The generalization of this method to multi- channel case is also developed and presented.

\section{Angular dependent continuum ambiguity}

Let us recall that observables in single-channel reactions are given as a sum of products involving one (helicity or transversity) amplitude with the complex conjugate of another, so that the general form of any observable is $\mathscr{O}=f\left(H_{k} \cdot H_{l}^{*}\right)$, where $f$ is a known, well-defined real function. The direct consequence is that any observable is invariant with respect to the following simultaneous phase transformation of all amplitudes:

$$
\begin{aligned}
H_{k}(W, \theta) \rightarrow \tilde{H}_{k}(W, \theta) & =e^{i \phi(W, \theta)} \cdot H_{k}(W, \theta) \\
\text { for all } k & =1, \cdots, n
\end{aligned}
$$

where $\mathrm{n}$ is the number of spin degrees of freedom ( $\mathrm{n}=1$ for the 1-dim toy model, $\mathrm{n}=2$ for pi-N scattering and $\mathrm{n}=4$ for pseudoscalar meson photoproduction), and $\phi(W, \theta)$ is an arbitrary, real function which is the same for all contributing amplitudes.

As resonance properties are usually the goal of such studies, and these are identified with poles of the partial-wave (or multipole) amplitudes, we must analyze the influence of the continuum ambiguity not upon helicity or transversity amplitudes, but upon their partial wave decompositions. To simplify the study we introduce partial waves in a simplified version than those found in Ref. [5]:

$$
A(W, \theta)=\sum_{\ell=0}^{\infty}(2 \ell+1) A_{\ell}(W) P_{\ell}(\cos \theta)
$$

where $A(W, \theta)$ is a generic notation for any amplitude $H_{k}(W, \theta), k=1, \cdots n$. The complete set of 
observables remains unchanged when we make the following transformation:

$$
\begin{aligned}
A(W, \theta) \rightarrow \tilde{A}(W, \theta) & =e^{i \phi(W, \theta)} \\
& \times \sum_{\ell=0}^{\infty}(2 \ell+1) A_{\ell}(W) P_{\ell}(\cos \theta) \\
\tilde{A}(W, \theta) & =\sum_{\ell=0}^{\infty}(2 \ell+1) \tilde{A}_{\ell}(W) P_{\ell}(\cos \theta)
\end{aligned}
$$

We are interested in rotated partial wave amplitudes $\tilde{A}_{\ell}(W)$, defined by Eq.(2.3), and are free to introduce the Legendre decomposition of an exponential function as:

$$
e^{i \phi(W, \theta)}=\sum_{\ell=0}^{\infty} L_{\ell}(W) P_{\ell}(\cos \theta) .
$$

After some manipulation of the product $P_{\ell}(x) P_{k}(x)$ (see refs. [6, 7] for details of the summation rearrangement) we obtain:

$$
\tilde{A}_{\ell}(W)=\sum_{\ell^{\prime}=0}^{\infty} L_{\ell^{\prime}}(W) \cdot \sum_{m=\left|\ell^{\prime}-\ell\right|}^{\ell^{\prime}+\ell}\left\langle\ell^{\prime}, 0 ; \ell, 0 \mid m, 0\right\rangle^{2} A_{m}(W)
$$

where $\left\langle\ell^{\prime}, 0 ; \ell, 0 \mid m, 0\right\rangle$ is a standard Clebsch-Gordan coefficient.

To get a better insight into the mechanism of multipole mixing, let us expand Eq. (2.5) in terms of phase-rotation Legendre coefficients $L_{\ell^{\prime}}(W)$ :

$$
\begin{aligned}
& \tilde{A}_{0}(W)=\mathbf{L}_{\mathbf{0}}(\mathbf{W}) \mathbf{A}_{\mathbf{0}}(\mathbf{W})+L_{1}(W) A_{1}(W)+L_{2}(W) A_{2}(W)+\ldots \\
& \tilde{A}_{1}(W)=\mathbf{L}_{\mathbf{0}}(\mathbf{W}) \mathbf{A}_{\mathbf{1}}(\mathbf{W})+L_{1}(W)\left[\frac{1}{3} A_{0}(W)+\frac{2}{3} A_{2}(W)\right]+L_{2}(W)\left[\frac{2}{5} A_{1}(W)+\frac{3}{5} A_{3}(W)\right]+\ldots \\
& \tilde{A}_{2}(W)=\mathbf{L}_{\mathbf{0}}(\mathbf{W}) \mathbf{A}_{2}(\mathbf{W})+L_{1}(W)\left[\frac{2}{5} A_{1}(W)+\frac{3}{5} A_{3}(W)\right]+L_{2}(W)\left[\frac{1}{5} A_{0}(W)+\frac{2}{7} A_{2}(W)+\frac{18}{35} A_{4}(W)\right]+\ldots
\end{aligned}
$$

The consequence of Eqs. (2.5) and (2.6) is that angular-dependent phase rotations mix multipoles.

\section{Conclusion:}

Without fixing the free continuum ambiguity phase $\phi(W, \theta)$, the partial wave decomposition $A_{\ell}(W)$ defined in Eq. (2.2) is non-unique. Partial waves get mixed, and identification of resonance quantum numbers might be changed. To compare different partial-wave analyses, it is essential to match the continuum ambiguity phase; otherwise the mixing of multipoles is yet another, uncontrolled, source of systematic errors. Observe that this phase rotation does not create new pole positions, but just reshuffles the existing ones among several partial waves.

\section{Using angular-dependent phase ambiguity to obtain up-to-a-phase unique, unconstrained, single-energy solution in $\eta$ photoproduction}

We perform unconstrained, $L_{\max }=5$ truncated single-energy analyses on a complete set of observables for $\eta$ photoproduction given in the form of pseudo-data created using the ETA-MAID15a 
model [8]: $d \sigma / d \Omega, \Sigma d \sigma / d \Omega, T d \sigma / d \Omega, F d \sigma / d \Omega, G d \sigma / d \Omega, P d \sigma / d \Omega, C_{x^{\prime}} d \sigma / d \Omega$, and $O_{x^{\prime}} d \sigma / d \Omega$. All higher multipoles are put to zero. The fitting procedure finds solutions which are non-unique, and we obtain many solutions depending on the choice of initial parameters in the fit. In Fig. 1 we show a complete set of pseudo-data with the error of $1 \%$ created at 18 angles (red symbols), and the typical SE fit (full line) at one representative energy of $W=1769.80 \mathrm{MeV}$.

Elab $=1200.00 \mathrm{MeV}, \mathrm{Wcm}=1769.80 \mathrm{MeV}$
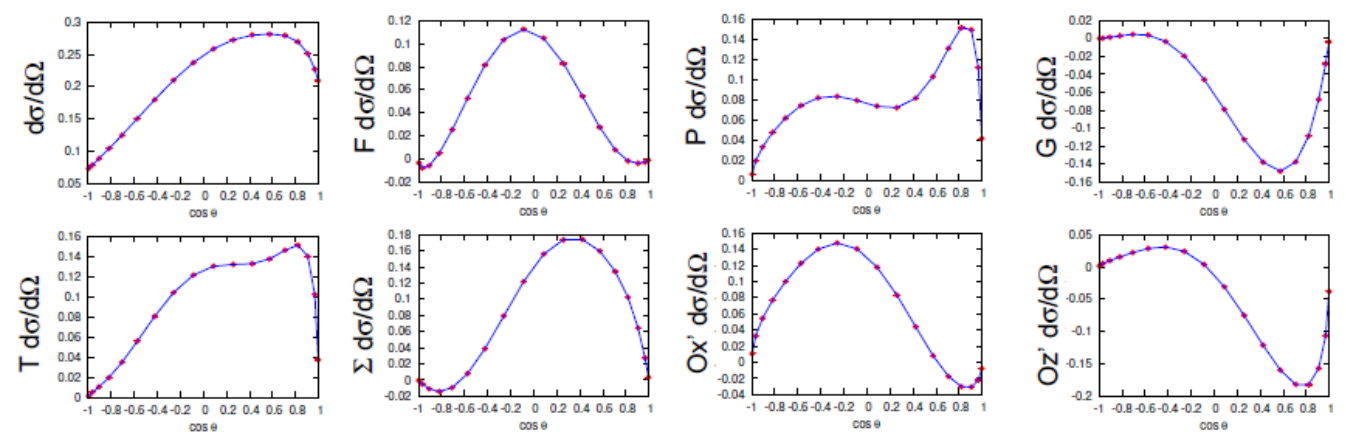

Figure 1: Complete set of observables for $\eta$ photoproduction given in the form of pseudo-data created at 18 angles with the error bar of $1 \%$ using the ETA-MAID15a model (red symbols) and a typical fit to the data (full line).

In Fig. 2 we show an example of three very different sets of multipoles which fit the complete pseudo-data set equally well to a high precision: two discrete and discontinuous ones obtained by setting the initial fitting values to the ETA-MAID16a [9] (SE $\left.{ }^{16 a}\right)$ and Bonn-Gatchina [10] (SE $\left.{ }^{B G}\right)$ model values (blue and red symbols respectively), and the generating ETA-MAID15a model [8] which is displayed as full and dashed black continuous lines.
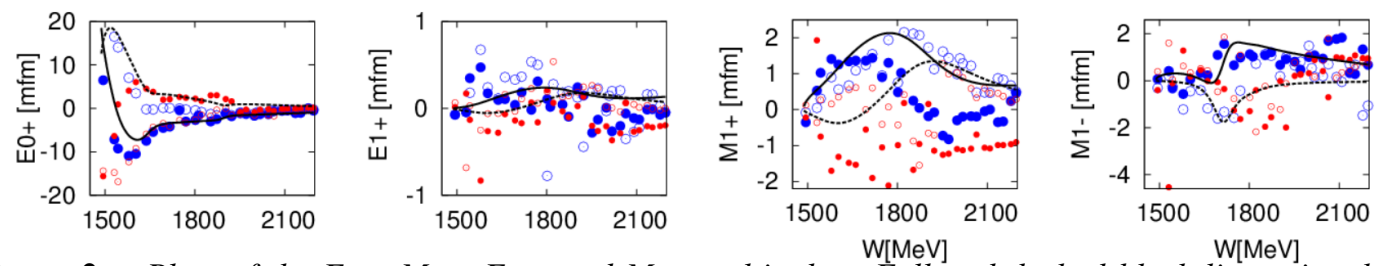

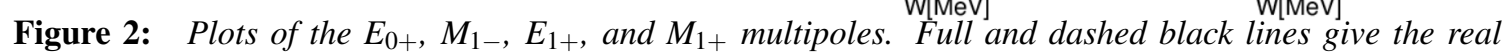
and imaginary part of the ETA-MAID15a generating model. Discrete blue and red symbols are obtained with the unconstrained, $L_{\max }=5$ fits of a complete set of observables generated as numeric data from the ETA-MAID15a model of ref. [8], with the initial fitting values taken from the ETA-MAID16a [9] and the Bonn-Gatchina [10] models respectively. Filled symbols represent the real parts and open symbols give the imaginary parts.

We know from Eq.(1) that equivalent fits to a complete set of data must be produced by helicity amplitudes with different phases. Therefore, in Fig. 3, we construct the helicity amplitudes corresponding to all three sets of multipoles from Fig. 2 at one randomly chosen energy $W=1660.4 \mathrm{MeV}$. 

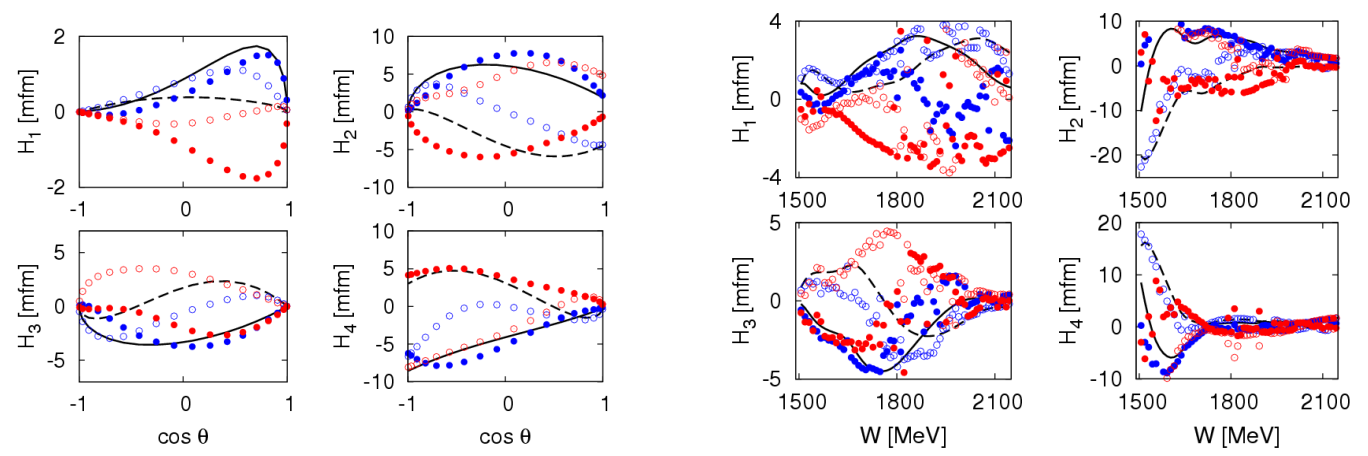

Figure 3: Left we show three sets of helicity amplitudes for all three sets of multipoles at one randomly chosen energy $W=1660.4 \mathrm{MeV}$, and right for we show the excitation curves for all three sets of multipoles, at one randomly chosen value of $\cos \theta=0.2588 \mathrm{MeV}$. The figure coding is the same as in Fig. 2.

We see that all three sets of helicity amplitudes are indeed different, but the discontinuity of multipole amplitudes, observed in Fig. 2-left is not reflected in a plot of helicity amplitudes at a fixed single energy. If instead we plot an excitation curve of all four helicity amplitudes at a randomly chosen angle, which is arbitrarily set to the value $\cos \theta=0.2588$, we obtain the result shown in Fig. 3-right.

We see that the excitation curve of helicity amplitudes in this case remains continuous only for the generating model ETA-MAID15a. For both single-energy solutions it is different, and at the same time shows notable discontinuities between neighbouring energy points. This leads to the following understanding of this, apparently very different multipole solutions:

When we perform an unconstrained SE PWA, each minimization is performed independently at individual energies, and the phase is chosen randomly. So, at each energy the fit chooses a different angle dependent phase, and creates different, discontinuous numerical values for each helicity amplitude, producing discontinuous sets of multipoles.

However, the invariance with respect to phase rotations offers a possible solution. Let us show the procedure.

We introduce the following angle-dependent phase rotation simultaneously for all four helicity amplitudes:

$$
\begin{aligned}
\tilde{H}_{k}^{S E}(W, \theta) & =H_{k}^{S E}(W, \theta) \cdot e^{i \Phi_{H_{2}}^{15 a}(W, \theta)-i \Phi_{H_{2}}^{S E}(W, \theta)} \\
k & =1, \ldots, 4
\end{aligned}
$$

where $\Phi_{H_{2}}^{S E}(W, \theta)$ is the phase of any single-energy solution and $\Phi_{H_{2}}^{15 a}(W, \theta)$ is the phase of generating solution ETA-MAID15a. Applying this rotation we replace the discontinuous $\Phi_{H_{2}}^{S E}(W, \theta)$ phase from any SE solution with the continuous $\Phi_{H_{2}}^{15 a}(W, \theta)$ ETA-MAID15a phase.

The resulting rotated single-energy helicity amplitudes are compared with generating ETAMAID15a amplitudes in Fig. 4.

We see that rotated helicity amplitudes of both single-energy solutions are now identical to the generating ETA-MAID15a helicity amplitudes.

Thus, the previously different sets of discrete, discontinuous single-energy multipoles different from the generating solution ETA-MAID15a and given in Fig. 2, are after phase rotation trans- 

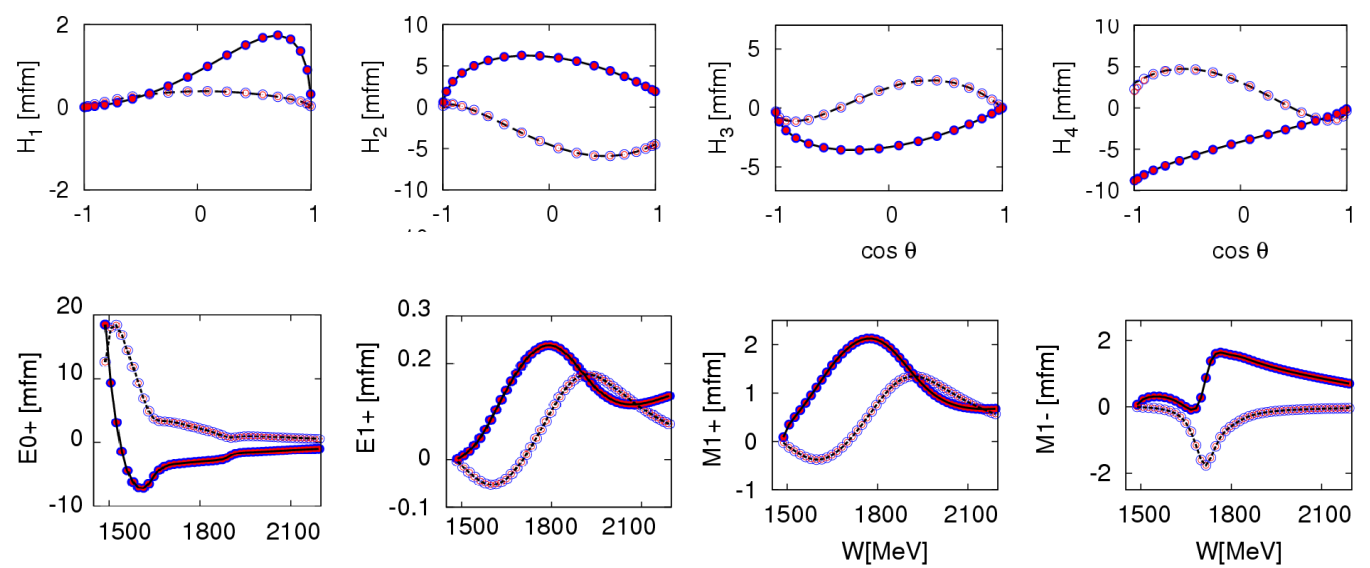

Figure 4: $U p$ we show all three sets of rotated helicity amplitudes at one randomly chosen energy $W=$ $1660.4 \mathrm{MeV}$, and down three sets of rotated multipoles. The figure coding is the same as in Fig. 2.

formed into continuous multipoles now identical to the generating solution, and given in lower part of Fig. 4.

So, we have constructed a way to generate up-to-a-phase unique solutions in an unconstrained PWA of a complete set of observables generated as pseudo-data.

\section{Laurent + Pietarinen expansion}

The driving concept behind the Laurent-Pietarinen $(\mathrm{L}+\mathrm{P})$ expansion was the aim to replace an elaborate theoretical model by a local power-series representation of partial wave amplitudes [2]. The complexity of a partial-wave analysis model is thus replaced by much simpler model-independent expansion which just exploits analyticity and unitarity. The L+P approach separates pole and regular part in the form of a Laurent expansion, and instead of modeling the regular part in some physical model it uses the conformal mapping to expand it into a rapidly converging power series with well defined analytic properties. So, the method replaces the regular part calculated in a model by the simplest analytic function which has correct analytic properties of the analyzed partial wave (multipole), and fits the data. In such an approach the model dependence is minimized, and is reduced to the choice of the number and location of branch-points used in the model.

The $\mathrm{L}+\mathrm{P}$ expansion is based on the Pietarinen expansion used in some former papers in the analysis of pion-nucleon scattering data $[11,12,13,14]$, but for the $\mathrm{L}+\mathrm{P}$ model the Pietarinen expansion is applied in a different manner. It exploits the Mittag-Leffler expansion ${ }^{1}$ of partial wave amplitudes near the real energy axis, representing the regular, but unknown, background term by a conformal-mapping-generated, rapidly converging power series called a Pietarinen expansion ${ }^{2}$. The method was used successfully in several few-body reactions $[3,4,17]$, and recently gener-

\footnotetext{
${ }^{1}$ Mittag-Leffler expansion [15]. This expansion is the generalization of a Laurent expansion to a more-than-one pole situation. For simplicity, we will simply refer to this as a Laurent expansion.

${ }^{2}$ A conformal mapping expansion of this particular type was introduced by Ciulli and Fisher [11, 12], was described in detail and used in pion-nucleon scattering by Esco Pietarinen [13, 14]. The procedure was denoted as a Pietarinen expansion by G. Höhler in [16].
} 
alized to the multi-channel case [18]. The formulae used in the $\mathrm{L}+\mathrm{P}$ approach are collected in Table 1. In the fits, the regular background part is represented by three Pietarinen expansion series,

Table 1: Formulae defining the Laurent+Pietarinen $(\mathrm{L}+\mathrm{P})$ expansion.

$$
\begin{aligned}
& T^{a}(W)=\quad \sum_{j=1}^{N_{\text {pole }}} \frac{x_{j}^{a}+\imath y_{j}^{a}}{W_{j}-W}+\sum_{k=0}^{K^{a}} c_{k}^{a} X^{a}(W)^{k}+\sum_{l=0}^{L^{a}} d_{l}^{a} Y^{a}(W)^{l}+\sum_{m=0}^{M^{a}} e_{m}^{a} Z^{a}(W)^{m} \\
& X^{a}(W)=\quad \frac{\alpha^{a}-\sqrt{x_{P}^{a}-W}}{\alpha^{a}+\sqrt{x_{P}^{a}-W}} ; \quad Y^{a}(W)=\frac{\beta^{a}-\sqrt{x_{Q}^{a}-W}}{\beta^{a}+\sqrt{x_{Q}^{a}-W}} ; \quad Z^{a}(W)=\frac{\gamma^{a}-\sqrt{x_{R}^{a}-W}}{\gamma^{a}+\sqrt{x_{R}^{a}-W}} \\
& D_{d p}^{a}=\frac{1}{2 N_{W}^{a}-N_{p a r}^{a}} \sum_{i=1}^{N_{W}^{a}}\left\{\left[\frac{\operatorname{Re} T^{a}\left(W^{(i)}\right)-\operatorname{Re} T^{a, \exp }\left(W^{(i)}\right)}{E r r_{i, a}^{\mathrm{Re}}}\right]^{2}+\left[\frac{\operatorname{Im} T^{a}\left(W^{(i)}\right)-\operatorname{Im} T^{a, \exp }\left(W^{(i)}\right)}{E r r_{i, a}^{\operatorname{Im}}}\right]^{2}\right\}+\mathscr{P}^{a} \\
& \mathscr{P}^{a}=\quad \lambda_{c}^{a} \sum_{k=1}^{K^{a}}\left(c_{k}^{a}\right)^{2} k^{3}+\lambda_{d}^{a} \sum_{l=1}^{L^{a}}\left(d_{l}^{a}\right)^{2} l^{3}+\lambda_{e}^{a} \sum_{m=1}^{M^{a}}\left(e_{m}^{a}\right)^{2} m^{3} \quad D_{d p}=\sum_{a}^{a l l} D_{d p}^{a} \\
& \text { a ..... channel index } \quad N_{\text {pole }} \ldots . . \text { number of poles } \quad W_{j}, W \in \mathbb{C} \\
& x_{i}^{a}, y_{i}^{a}, c_{k}^{a}, d_{l}^{a}, e_{m}^{a}, \alpha^{a}, \beta^{a}, \gamma^{a} \ldots \in \mathbb{R} \\
& K^{a}, L^{a}, M^{a} \ldots \in \mathbb{N} \text { number of Pietarinen coefficients in channel } a \text {. } \\
& D_{d p}^{a} \ldots . . \text { discrepancy function in channel } a \quad N_{W}^{a} \ldots . . \text { number of energies in channel } a \\
& N_{\text {par }}^{a} \text {...... number of fitting parameters in channel } a \quad \mathscr{P}^{a} \text {..... Pietarinen penalty function } \\
& \lambda_{c}^{a}, \lambda_{d}^{a}, \lambda_{e}^{a} \ldots . . \text { Pietarinen weighting factors } \quad x_{P}^{a}, x_{Q}^{a}, x_{R}^{a} \in \mathbb{R}(\text { or } \in \mathbb{C}) \text {. } \\
& \operatorname{Err}_{i, a}^{\mathrm{Re}, \operatorname{Im}} \ldots . . . \text { minimization error of real and imaginary part respectively. }
\end{aligned}
$$

all free parameters are fitted. The first Pietarinen expansion with branch-point $x_{P}$ is restricted to an unphysical energy range and represents all left-hand cut contributions. The next two Pietarinen expansions describe the background in the physical range with branch-points $x_{Q}$ and $x_{R}$ respecting the analytic properties of the analyzed partial wave. The second branch-point is mostly fixed to the elastic channel branch-point, the third one is either fixed to the dominant channel threshold, or left free. Thus, only rather general physical assumptions about the analytic properties are made like the number of poles and the number and the position of branch-points, and the simplest analytic function with a set of poles and branch-points is constructed. The method is applicable to both, theoretical and experimental input, and represents the first reliable procedure to extract pole positions from experimental data, with minimal model bias.

The generalization of the $\mathrm{L}+\mathrm{P}$ method to a multichannel $\mathrm{L}+\mathrm{P}$ method is performed in the following way: i) separate Laurent expansions are made for each channel; ii) pole positions are fixed for all channels, iii) residua and Pietarinen coefficients are varied freely; iv) branch-points are chosen as for the single-channel model; v) the single-channel discrepancy function $D_{d p}^{a}$ (see Eq. (5) in ref. [17]) which quantifies the deviation of the fitted function from the input is generalized to a multi-channel quantity $D_{d p}$ by summing up all single-channel contributions, and vi) the minimization is performed for all channels in order to obtain the final solution.

The formulae used in the $\mathrm{L}+\mathrm{P}$ approach are collected in Table 1. 


\section{Acknowledgements}

This work was supported by the Deutsche Forschungsgemeinschaft SFB 1044.

\section{References}

[1] A. Svarc, https://indico.cern.ch/event/591374/contributions/2477135/, PWA9/ATHOS4: The International Workshop on Partial Wave Analyses and Advanced Tools for Hadron Spectroscopy, Bad Honnef near Bonn (Germany) from March 13 to 17, 2017.

[2] A. Svarc, M. Hadzimehmedovic, H. Osmanovic, J. Stahov, L. Tiator, and R. L. Workman, Phys, Rev. C88, 035206 (2013).

[3] A. Svarc, M. Hadzimehmedovic, R. Omerovic, H. Osmanovic, and J. Stahov, Phys, Rev. C89, 0452205 (2014).

[4] A. Svarc, M. Hadzimehmedovic, H. Osmanovic, J. Stahov, L. Tiator, and R. L. Workman, Phys, Rev. C89, 65208 (2014).

[5] L. Tiator, D. Drechsel, S. S. Kamalov and M. Vanderhaeghen, Eur. Phys. J. ST 198, 141 (2011).

[6] J. Dougall, Glasgow Mathematical Journal, 1 (1952) 121-125.

[7] Y. Wunderlich, A. Švarc, R. L. Workman, L. Tiator, and R. Beck, arXiv:1708.06840[nucl-th].

[8] V. L. Kashevarov, L. Tiator, M. Ostrick, Bled Workshops Phys., 16, 9 (2015).

[9] V. L. Kashevarov, 1. Tiator, M. Ostrick, JPS Conf. Proc. 13, 020029 (2017).

[10] http://pwa.hiskp.uni-bonn.de/

[11] S. Ciulli and J. Fischer in Nucl. Phys. 24, 465 (1961).

[12] I. Ciulli, S. Ciulli, and J. Fisher, Nuovo Cimento 23, 1129 (1962).

[13] E. Pietarinen, Nuovo Cimento Soc. Ital. Fis. 12A, 522 (1972).

[14] E. Pietarinen, Nucl. Phys. B107, 21 (1976).

[15] Michiel Hazewinkel: Encyclopaedia of Mathematics, Vol.6, Springer, 31. 8. 1990, pg.251.

[16] G. Höhler and H. Schopper, "Numerical Data And Functional Relationships In Science And Technology. Group I: Nuclear And Particle Physics. Vol. 9: Elastic And Charge Exchange Scattering Of Elementary Particles. B: Pion Nucleon Scattering. Pt. 2: Methods And Results and Phenomenology," Berlin, Germany: Springer ( 1983) 601 P. ( Landolt-Boernstein. New Series, I/9B2).

[17] A. Š, M. Hadžimehmedović, H. Osmanović, J. Stahov, and R. L. Workman, Phys. Rev. C91, 015207 (2015).

[18] A. Š, M. Hadžimehmedović, H. Osmanović, J. Stahov, L. Tiator, R. L. Workman, Phys. Lett. B755 (2016) 452âĂŞ455. 